\title{
Metástasis duodenal de cáncer de mama. Presentación atípica y resolución quirúrgica no habitual
}

\author{
Roberto Muñoz Jaramillo, Sonia Ojeda, Tomás Fumo, Lucio Uranga, Oscar Zerbo, Vanesa Mikolaitis, \\ Rodolfo Corti
}

Hospital de Gastroenterología Dr. Carlos Bonorino Udaondo. Ciudad Autónoma de Buenos Aires, Argentina.

Acta Gastroenterol Latinoam 2020;50(2):150-153

Recibido: 02/08/2018 / Aceptado: 24/07/2019 / Publicado online: 29/06/2020

\section{Resumen}

El cáncer de mama es el tumor maligno más frecuente y la principal causa de muerte por cáncer en el mundo. Los sitios más frecuentes de metástasis son el hueso, el higado y el pulmón y menos del 1\% compromete el tracto gastrointestinal. Se presenta el caso clínico de una paciente con antecedentes de cáncer de mama tratada con quimioterapia y metástasis duodenal. Se le realizó duodenopancreatectomía cefálica (DPC) y quimioterapia adyuvante con una evolución favorable.

Palabras claves. Neoplasias de la mama, duodeno, metástasis de la neoplasia.

Correspondencia: Sonia Ojeda

Tucumán 1924, $3^{\circ}$ A (C.P.: 1050). Ciudad Autónoma de Buenos Aires, Argentina

Tel.: 011-60277698

Correo electrónico: soniaojeda11@gmail.com

\section{Duodenal metastasis of breast cancer. Atypical presentation and unusual surgical resolution}

\section{Summary}

Breast cancer is the most frequent malignant tumor and the leading cause of death worldwide. The most frequent sites of metastatic involvement are the bone, liver and lung, and less than 1\% compromise the gastrointestinal tract. The case of a patient with a history of breast cancer treated with chemotherapy and subsequent duodenal relapse is presented below. A cephalic duodenopancreatectomy (CDP) and adjuvant chemotherapy were performed with favorable evolution.

Key words. Breast neoplasms, duodenum, neoplasm metastasis.

\author{
Abreviaturas \\ DPC: Duodenopancreatectomía cefálica. \\ TC: Tomografia computada. \\ GI: Gastrointestinal.
}

\section{Introducción}

El cáncer de mama es el tumor maligno más frecuente en Argentina, con un promedio de 19000 casos diagnosticados por año. ${ }^{1}$ Representa el $17 \%$ de todos los tumores malignos y cerca de un tercio de los cánceres en la mujer. El compromiso metastásico del cáncer de mama involu- 
cra a los pulmones, los huesos, el cerebro y al hígado, siendo infrecuente la afectación gastrointestinal. ${ }^{2,4}$ Presentamos el caso de una paciente con cáncer de mama y metástasis duodenal con el objetivo de destacar la importancia de considerar al cáncer de mama metastásico como una causa de afectación gastrointestinal, especialmente en mujeres con dicho antecedente.

\section{Caso clínico}

Una paciente de sexo femenino de 34 años de edad consultó por náuseas, epigastralgia y plenitud postprandial de dos meses de evolución. Como antecedente significativo presentó un carcinoma invasor de mama de tipo no especificado y carcinoma ductal in situ de bajo grado sin invasión linfovascular ni perineural dos años previos a la consulta (T2N2M0 -Estadio 3). Se le realizó quimioterapia con esquema neoadyuvante con adriamicina, ciclofosfamida, mastectomía simple con vaciamiento axilar completo y, posteriormente, adyuvancia con docetaxel y radioterapia. El examen físico y los parámetros de laboratorio no demostraron alteraciones.

Se le realizó una videoendoscopía digestiva alta la cual evidenció en el duodeno la presencia de un nicho excavado de aproximadamente $1,5 \mathrm{~cm}$ con fondo de fibrina de bordes netos regulares. La anatomía patológica describió proliferación neoplásica de células epiteliales con núcleos redondeados relativamente uniformes, distribuidas en nidos y ductos.

La tomografía computada (TC) evidenció una imagen nodular de 30 x $43 \mathrm{~mm}$ en la cara interna y superior de la segunda porción duodenal con aspecto neoformativo (Figura 1). Se realizó un centellograma sin alteraciones.

Figura 1. Imagen nodular de $30 \times 43 \mathrm{~mm}$ de la cara interna y superior de la segunda porción duodenal con aspecto neoformativo en la TC.



Se arribó al diagnóstico de una infiltración duodenal por carcinoma ductal de origen mamario.

En el Comité Interdisciplinario se decidió realizar una laparoscopía estadificadora por ser la metástasis intestinal, en ausencia de compromiso locorregional, un indicador de mayor supervivencia. En el acto quirúrgico se evidenció una lesión de $5 \mathrm{~cm}$ al nivel de la rodilla duodenal superior con extensión medial, sin plano de clivaje con la cabeza pancreática y extensión retro portal. Por esto se realizó una DPC laparoscópica (Figuras $2 \mathrm{a}$ y 2b). La anatomía patológica de la pieza fue coincidente con la biopsia endoscópica y la inmunohistoquímica fue CK7 positivo, CK20 negativo, con receptores de estrógenos y progesterona positivos, GCDFP15 negativo, Cdx2 negativo (Figura 3). La evolución posoperatoria fue favorable. Realizó quimioterapia adyuvante con una buena tolerancia y respuesta.

Figura 2. A) Pieza quirúrgica. B) Pieza quirúrgica. Formación tumoral nodular de $5 \times 4,5 \times 4 \mathrm{~cm}$.
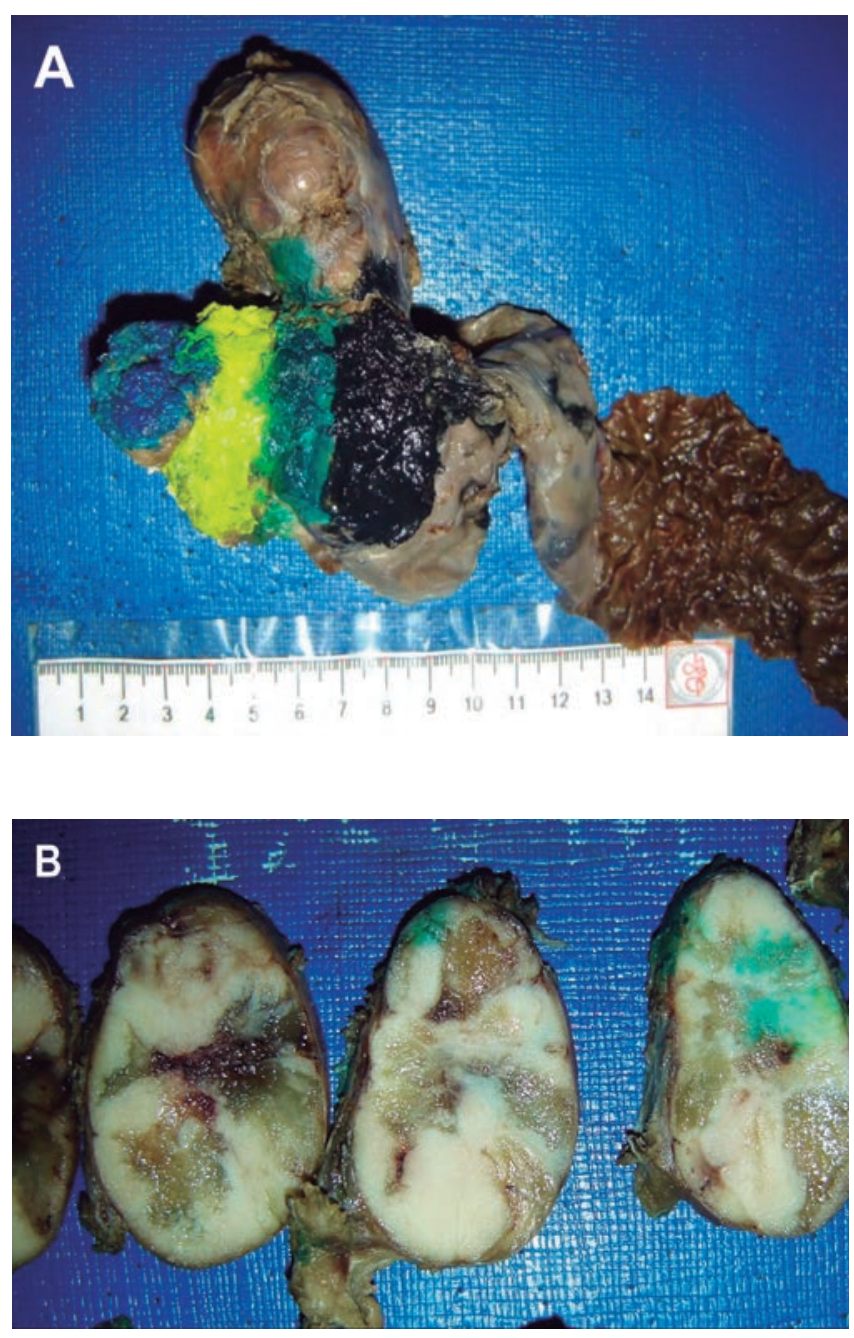
Figura 3. Anatomía patológica coincidente con infiltración duodenal por carcinoma mamario.



\section{Discusión}

Las metástasis gastrointestinales del cáncer de mama son infrecuentes. Un estudio de necropsia demostró que la afectación gastrointestinal (GI) varía del $6 \%$ al $18 \%$, presentándose mayormente con el subtipo lobular. ${ }^{2} \mathrm{Un}$ estudio retrospectivo demuestra que los sitios de afectación involucran principalmente el colon y el recto (48\%), seguido del estómago (28\%) y del intestino delgado (19\%). La presentación clínica es inespecífica y se estima que el intervalo entre el diagnóstico primario y la aparición de las metástasis es de 7 años, ${ }^{3}$ aunque otras revisiones determinan su aparición hasta 20 años después del diagnóstico inicial. ${ }^{4}$

Existen escasos reportes en la literatura sobre el cáncer de mama con metástasis en el intestino delgado. Khairy et al. describen el caso de una paciente que presentó obstrucción duodenal como manifestación inicial de un cáncer de mama metastásico. ${ }^{5}$ Frase-Moodie et al. reportan en el año 1974 el caso de una paciente, con diagnóstico de cáncer de mama, que presentó 13 años después una metástasis en yeyuno, que fue tratada quirúrgicamente con una evolución favorable, ${ }^{6} \mathrm{y}$ un caso similar con metástasis ileal fue asimismo descripto. ${ }^{8}$ Otros reportes demuestran el compromiso duodenal en el contexto de una enfermedad metastásica avanzada con pobre respuesta al tratamiento paliativo. . $^{\text {7-9 }}$

Es de destacar en el caso descripto que la paciente presentó una enfermedad metastásica localizada. Inicialmente se consideró una terapia con intención curativa, realizándose una DPC con una evolución favorable. No existen reportes previos de esta cirugía por metástasis de cáncer mamario. La respuesta oncológica de las metástasis del carcinoma ductal de mama difiere según el órgano comprometido. El compromiso del tracto digestivo suele tener una pobre repuesta a la quimioterapia, por lo que la resección quirúrgica es la opción terapéutica en metás- 
tasis únicas o como paliación de lesiones sintomáticas.

Mantener un alto grado de sospecha puede evitar el diagnóstico tardío y resultar en un tratamiento temprano que mejore el pronóstico. Debido al aumento en la sobrevida del cáncer de mama por la mejora en los regímenes terapéuticos se debe tener presente la metástasis en sitios inusuales, inclusive si los síntomas aparecen muchos años después.

Sostén financiero. No se ha recibido apoyo financiero para la realización del presente manuscrito.

\section{Referencias}

1. Ballesteros I, Limardo L, Abriata MG. Análisis de situación por cáncer en Argentina. Boletín vigilancia epidemiológica 2018.

2. Amin A, Reddy A, Jha M, Prasad K. Rectal metastasis from breast cancer: an interval of 17 years. BMJ Case Reports 2011; 96 (17): 1-3.
3. McLemore E, Pockaj B, Reynolds C, Gray R, Hernandez J, Grant $\mathrm{C}$, Donohue J. Breast cancer: presentation and intervention in women with gastrointestinal metastasis and carcinomatosis. Ann Surg Oncol 2005; 12 (11): 886-894.

4. Nazareno J, Taves D, Preiksaitis H. Metastatic breast cancer to the gastrointestinal tract: A case series and review of the literature. World J Gastroenterol 2006; 12 (38): 6219-6224.

5. Khairy S, Azzam A, Mohammed S, Suleman K, Khawaji A, Amin T. Duodenal obstruction as first presentation of metastatic breast cancer. Case Rep Surg 2015; 2015: 1-4.

6. Fraser-Moodie A, Burn I. Small-bowel metastases from carcinoma of the breast. Proc R Soc Med 1974; 67 (10): 1023-1024.

7. Choi J, Park S, Jeon M, Kang S, Lee S, Bae Y, Kim M. Solitary Small Bowel Metastasis from Breast Cancer. J Breast Cancer 2011; 14 (1): 69-71.

8. Shrestha S, Shah B, Tandukar S. Duodenal obstruction as the presenting manifestation of recurrent breast cancer. J Cancer Res Ther 2014; 10 (3): 761-762.

9. Rego R, Atiq M, Velchala N, Nevin D, McElreath D, McKnight W, Aduli F. Ampullary metastasis from breast cancer: an unusual finding. GE Port J Gastroenterol 2016; 23 (6): 300-303. 